\title{
Stratification of, albeit Artificial Intelligent (Al) Driven, High-Risk Elderly Outpatients for priority house call visits - a framework to transform healthcare services from reactive to preventive
}

\author{
Chandrasekar Vuppalapati ${ }^{1, *}$, Anitha Ilapakurti ${ }^{2}$, Santosh Kedari3 and Jayashankar Vuppalapati ${ }^{4}$ \\ ${ }^{1}$ Hanumayamma Innovations and Technologies, Inc., Senior Vice President, Fremont, California, USA 94536 \\ ${ }^{2}$ CEO, Hanumayamma Innovations and Technologies, Inc., 3 Product Manager, Hanumayamma Innovations and Technologies Private \\ Limited, 4 CTO, Hanumayamma Innovations and Technologies Private Limited
}

\begin{abstract}
House calls have nostalgic view and have practiced decades ago when the doctor arrived at the patient's door carrying a big black bag. House calls could prove to be a better way of treating very sick, elderly patients while they can still live at home. One of the greatest benefits is avoidance of Healthcare associated infections. Additionally, house calls save time and energy of immediate care members of and helps seek for ways to have transport for elderly. A house calls doctor, nonetheless, can see only five to seven patients a day. One reason is that a house call visit can take longer than an office visit, even after taking travel time into account. One way of optimizing house call delivery services is to employ AI based system to identify and generate priority list so that the healthcare providers have greater coverage of their needed patients house calls are performed in-time. In this paper, we propose innovative novel idea "AI enabled house calls are best medicine practices for the next generation". Finally, as part of the paper, we will present Sanjeevani house call service that is been deployed and currently in production
\end{abstract}

\section{Introduction}

House calls play an important role in improving healthcare delivery and patient outcomes, though sentimental view many link house call when the doctor arrived at the patient's door carrying a big black baga[1]. House calls could prove to be a better way of treating very sick, elderly patients while they can still live at home. In recent pilot program conducted in Oregon, Housecall Providers of Portland, Oregon, which had been operating at a loss, saved Medicare an average of almost $\$ 13,600$ for each patient in the pilot project. Its share of the savings was $\$ 1.2$ million. The house calls practice at MedStar Washington Hospital in Washington, D.C., cut the cost of care an average of $\$ 12,000$ per patient [1].

In rural communities, house call is thin border between staying versus relocating to the nearest urban settling, albeit forced relocation. A 30-minute house call also can save a patient's spouse or adult children from taking half a day off work to get a patient to my office [2].

Given very low immunity system, many senior citizens outpatients can avoid healthcare associated infections in Housecall settings. Health care-associated infections (HACI), or infections acquired in health-care settings are the most frequent adverse event in healthcare delivery worldwide [3]. Hundreds of millions of patients are affected by HCAI worldwide each year, leading to significant mortality and financial losses for health systems [3]. Of every 100 hospitalized patients at

\footnotetext{
${ }^{a}$ Doctors are saving money for Medicare via house calls https://www.healthcareitnews.com/news/doctors-are-saving-moneymedicare-house-calls
}

any given time, 7 in developed and 10 in developing countries will acquire at least one HCAI [3]. HCAIs account for 2 million cases and about 80,000 deaths a year in developing countries.

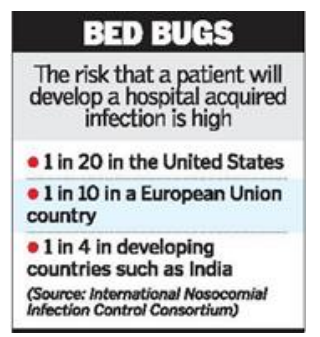

Fig. 1. Bed Bugs

A house calls doctor, nonetheless, can see only five to seven patients a day. One reason is that a house call visit can take longer than an office visit, even after taking travel time into account.

One way of optimizing house call delivery services is to employ Artificial Intelligence (AI) based system to identify and generate priority list so that the healthcare providers have greater coverage of their needed patients house calls are performed in-time. In this paper, we propose innovative novel idea "AI enabled house calls are best medicine practices for the next generation". Finally, as part of the paper, we will present Sanjeevani house call service that is been deployed

2 UNDERSTANDING ANALYTICS FOR stratification of OUTPATIENT DATA DRIVEN HOUSE CALLS

* Corresponding author: cvuppalapati@hanuinnotech.com 


\subsection{Life Cycle for Developing Machine Learning Process}

The Life Cycle for developing Machine Learning (ML) Process involves: Feature Engineering, Model Engineering and Model Evaluation (please see Fig, 2). Additionally, the development for ML is different from that of Software application because of the requirements to monitor and tune ML models in short iterations [Gartner].

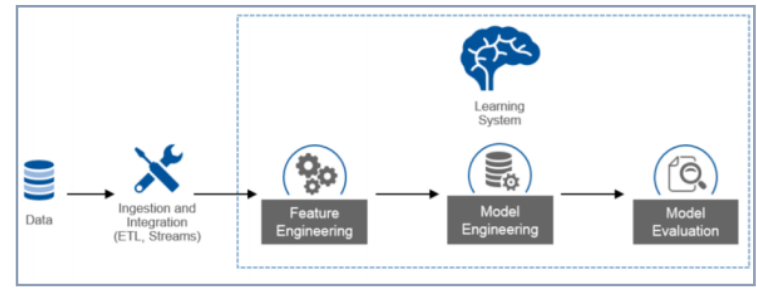

Fig. 2. Life Cycle for developing learning system

\subsection{Machine Learning}

Decision Tree: Decision tree algorithm is used to classify the attributes and select the outcome of the class attribute (Fig. 3). To construct decision tree both class attribute and item attributes are required. Decision tree is a tree like structure where the intermediate nodes represent attributes of the data, leaf nodes represents the outcome of the data and the branches hold the attribute value. Decision trees are widely used in the classification process because no domain knowledge is needed to construct the decision tree. Following figure shows simple decision trees [6], [7].

\subsubsection{Decision Tree}

The primary step in the decision tree algorithm is to identify the root node for the given set of data. Multiple methods exist to decide the root node of the decision tree. Information gain and Gini impurity are the primary methods used to identify the root node. Root node plays an important role in deciding which side of decision tree the data falls into. Like every classification methods, decision trees are also constructed using the training data and tested with the test data [19].

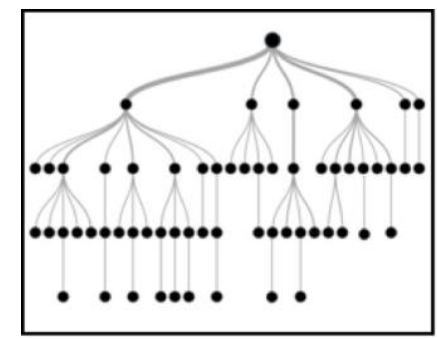

Fig. 3. Decision Tree

Information Gain: Information gain is used to the root node and the branch nodes in the decision tree. Information gain is calculated using entropy and information. The purpose of the information gain calculation is to identify the node that has least randomness or impurity. Entropy is calculated using the following formula [11], [19].

$\operatorname{Info}(D)=-\sum_{i=1}^{m} p i \log _{2}(p i)$

Information of the attribute is calculated using the following formula:

$$
\begin{gathered}
\operatorname{Info}_{A}(D)=\sum_{j=1}^{v} \frac{|D j|}{|D|} x \operatorname{info}(D j) \\
\operatorname{Gain}_{(A)}=\operatorname{Info}(D)-\operatorname{Info}_{A}(D)
\end{gathered}
$$

Information gain of an attribute is the difference between entropy and the information of that attribute. The attribute with the highest information gain is the root node, and the next level nodes are identified using the next high information gain attributes [11], [19].

\subsubsection{Naïve Bayesian Classifier}

A naive Bayes classifier (NBC) is a simple probabilistic classifier created by applying Bayes' theorem. To simplify computation, this classifier utilizes the assumption that data attributes are conditionally independent given the class value (activity label). Consider the problem of estimating the conditional probability distribution of activity labels $\mathrm{Y}$ over observed features X (i.e., $\mathrm{P}(\mathrm{Y} \mid \mathrm{X})$. By applying Bayes' rule, the conditional probability can be estimated as:

$$
\mathrm{P}(\mathrm{Y} \mid \mathrm{X})=\frac{P(X \mid Y) P(Y)}{P(X)}
$$

In the above equation, $\mathrm{P}(\mathrm{Y} \mid \mathrm{X})$, expresses the likelihood of observing a set of feature values for a given activity label, $\mathrm{P}(\mathrm{Y})$ represents the prior probability distribution over class values before observing the evidence, and $\mathrm{P}(\mathrm{X})$ represents the evidence, or the probability of observing the particular feature vector in the dataset. Place the figure as close as possible after the point where it is first referenced in the text. If there is a large number of figures and tables it might be necessary to place some before their text citation. If a figure or table is too large to fit into one column, it can be centred across both columns at the top or the bottom of the page.

\section{System Overview}

Sanjeevani Electronic Health Records provide electronic data storage and health analytics to the Users. Sanjeevani is an electronic healthcare data company that epitomizes provision of highly scalable EHR to the 
Users in Indian subcontinent. As part of enabling preventive and prognosis healthcare services, Sanjeevani exposed medical self-image analysis computer vision cloud (CV) (please see Figure 4). We have trained healthcare datasets related disease identification and skin rashes. Please note only subset of the images are being trained.

\section{A. Data Collection}

Sanjeevani Electronic Healthcare services conducts house calls and senior citizen services. Additionally, conducts health camps. Sanjeevani collects medical images as part of the above-mentioned services.

Additionally, Sanjeevani extracted Oncology image data from AAO. The AAO images are feature extracted and stored in LBP Reference Database.

\section{B. Feature Extraction}

Machine Learning feature extraction contains following steps: as shown in the below figure 4 , the image is first converted to monographic image, next extracted LBP vector, next constructed histogram. The histogram is translated into 20 bins 0 to 256-pixel value matrix. Next, average pixel value to construct unique array. Finally calculated unique feature for each image for classification purposes.

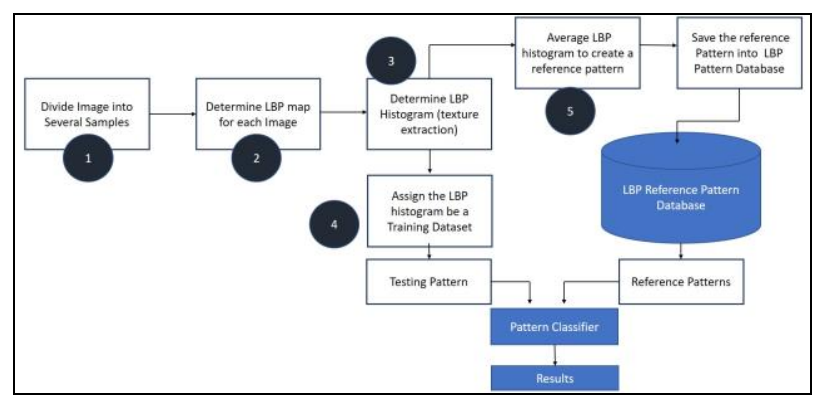

Fig. 4. Feature Extraction

\section{Pattern Database}

The pattern database (see figure 5) contains all the columns that are necessary to store texture features of the image and the data that can be fed to pattern classifier.

Some of the important columns include:

- LBP Histogram matrix

- Pattern (average values of histogram matrix)

- Unique Features per image

- UUID of the Image

- Original Image

- Monographic

- Histogram

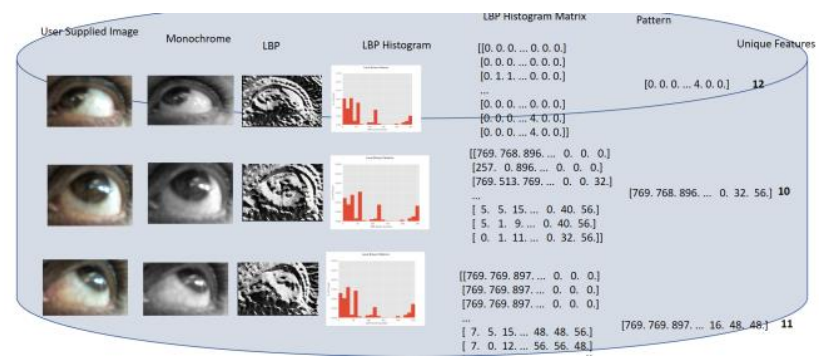

Figure 5: LBP Feature Extraction for Training dataset

The pattern database contains all the columns that are necessary to store texture features of the image and the data that can be fed to pattern classifier

\section{CONCLUSION AND FUTURE WORK}

This paper presented a novel and radical approach to integrate stratification models to attend House Call Visits. We staunchly believe that House Call visits greatly helps the health outcomes for elderly outpatients. We will continue expanding our input data sources and medical specialties to expand footprint of our AI cloud and to increase accuracy rates of our predictions and deliver precision House call services.

\section{References}

1. Kaiser Health News, Doctors are saving money for Medicare via house calls, May 23, 2016, https://www.healthcareitnews.com/news/doctorsare-saving-money-medicare-house-calls

2. Beth Oller, House Calls Improve Care, Lower Costs, January 28, 205, https:/www.aafp.org/news/blogs/freshperspectives/ entry/house calls improve care lower.html

3. WHO, Patient Safety - Health care-associated infections

FACT SHEET,

URL: http://www.who.int/gpsc/country work/gpsc ccisc fact sheet en.pdf, access date: August 6, 2018 4. Cinthya Anand, "When hospitals infect you",
February 2017 , https://www.thehindu.com/sci-tech/health/Whenhospitals-infect-you/article17289370.ece, Access date: August 6, 2018

5. Jiawei Han ,Micheline Kamber and Jian Pei, Data Mining: Concepts and Techniques, Morgan Kaufmann; 3 edition (July 6, 2011)

6. Anand Rajaraman and Jeffrey David Ullman , Mining of Massive Datasets, Cambridge University Press (December 30, 2011)

7. Chandrasekar Vuppalapati, Anaitha Ilapakurti, Santosh Kedari, "The Role of Big Data in Creating Sense EHR, an Integrated Approach to Create Next Generation Mobile Sensor and Wearable Data Driven Electronic Health Record (EHR)", Proceedings of INTERNATIONAL The Second SERVICE APPLICATIONS, 2016 IEEE Second International Conference on Big Data Computing Service and Applications (BigData) 